\title{
Reduction of Anyons to One Dimension and Calogero-Sutherland-type Models
}

\author{
Radhika Vathsan * \\ The Institute of Mathematical Sciences, \\ C.I.T. Campus, Taramani, Chennai 600 113, INDIA.
}

September $3 ! 997$

\begin{abstract}
The two-dimensional anyon system, when reduced to one dimension, yields models related to the Calogero-Sutherland model. One such reduction leads to a new model with a class of exact solutions. This model is one of a family of models obtained upon dimensional reduction of spherically symmetric models in arbitrary dimensions.
\end{abstract}

PACS No.: 03.65.-w

*email: radhika@imsc.ernet.in. 


\section{Introduction}

Anyons [1], as is well known now, are particles in two space dimensions with fractional exchange statistics. The anyon system is an example of quantum mechanics on multiply connected spaces and has wave functions that are multi-valued. A gauge transformation can be applied to trade this multi-valuedness for a specific potential, the so-called "statistical interaction". Thus a system of anyons can be treated as interacting particles (e.g. fermions ) with single-valued wave functions.

In one dimension, the Calogero-Sutherland model model ([3], [4]) is an example of a system exhibiting fractional exclusion statistics [0], in the sense proposed by Haldane [8]. There has been considerable speculation and interest in the connection between the anyon system and the Calogero-Sutherland (referred to henceforth as CSM), especially in regard to the connection between exchange and exclusion statistics (see, for instance, 91). Ideas of fractional exchange statistics in one dimension have been studied [7], and so have field theoretic models [10]. Dimensional reduction of the anyon system is a way of studying this connection [6]. These works are aimed at understanding the nature of statistics in one dimension and the approach taken is to arrive at a one-dimensional model with anyonic features, taking hints from the two-dimensional case of anyons.

Reducing the dimensions of the anyon system model could bear interesting relations to the CSM. Also, one could examine the possibility of obtaining exact solutions for the N-anyon problem (the non-linearly interpolating solutions) from possible integrable models obtained by such reduction.

The present work takes a different approach to the process of "reduction", by which is meant reducing the number of dimensions in the eigenvalue equation for the Hamiltonian of the system. This way, one ensures that the wave functions themselves reduce to those of the derived model. The main motivation behind this is that since we are within the realms of the original model itself, we can now try to deduce exact solutions for the two-dimensional anyon system, in the non-linearly interpolating regime. As it turns out, this cannot be achieved because of the peculiar restrictions under which such a dimensional reduction is possible.

Reduction to one dimension can be carried out in two ways: in polar coordinates, one can either freeze the radial coordinates to arrrive at a model defined on a circle, 
or freeze the angular coordinates to get a model on a (half-)line. In this paper we examine both these cases.

We first set the stage in section 2, describing the anyon system and fixing notations.

The case of reduction to the circle forms section 3. This has been considered in Ref. [11] but the resulting model is not a reduced anyon model. They arrive at the Sutherland model after a naive reduction by fixing one set of coordinates. However, to ensure that one is still within the realms of the same physical model, one must impose the restriction of coordinates on the whole eigenvalue equation, i.e on the wave functions after the derivatives are evaluated. Then one finds that the original eigenvalue equation ceases to be an eigenvalue equation. Impositions of restrictions on the radial behaviour of the wave functions yields inhomogeneous terms in the equation, that must be dealt with in order that the reduced equation become an eigenvalue equation.

The reduction to a line is considered in section 4 . Here, direct reduction yields a model which has no solutions derivable from the anyon system because of the restriction on the angular dependence of the wave functions. However, this model is of interest in that it can be obtained by the reduction of a spherically symmetric problem in arbitrary dimensions, with three-body interactions of the CSM type. In restricting to models with anyonic solutions, we find that we must first extract the

factor $\Pi_{i} \rho_{i}^{-1 / 2}$ which is the characteristic of anyonic behaviour and then proceed to fix the angular coordinates. In this case we obtain a model related to the CSM which has a class of exact solutions derived from the original anyon solutions.

We conclude with a summary and discussion of results.

\section{The Anyon System}

For $\mathrm{N}$ anyons in a plane in an oscillator potential, the Hamiltonian is, in terms of dimensionless quantities,

$$
H=\frac{1}{2} \sum_{i=1}^{N}\left[\left(\mathbf{p}_{i}-\mathbf{a}_{i}\right)^{2}+\mathbf{r}_{i}^{2}\right]
$$


where the statistics, contained in the parameter $\alpha$, has been incorporated in terms of a singular gauge potential

$$
\mathbf{a}_{i}=\alpha \hat{\mathbf{z}} \times \sum_{j \neq i} \frac{\mathbf{r}_{i j}}{r_{i j}^{2}}
$$

(The system is confined by an oscillator potential in order ensure a discrete spectrum.) Putting in $\mathbf{p}_{i}=-\iota \nabla_{i}$ and expanding,

$$
H=-\frac{1}{2} \sum_{i=1}^{N}\left(\nabla_{i}^{2}-r_{i}^{2}\right)+\iota \alpha \sum_{i<j=1}^{N} \frac{l_{i j}}{r_{i j}^{2}}+\frac{\alpha^{2}}{2} \sum_{i \neq j, k=1}^{N} \frac{\mathbf{r}_{i j} \cdot \mathbf{r}_{i k}}{r_{i j}^{2} r_{i k}^{2}}
$$

where $l_{i j}=\mathbf{r}_{i j} \times \nabla_{i j}$.

For our purpose it is more convenient to work in polar coordinates,

$$
x_{i}+\iota y_{i}=\rho_{i} e^{\iota \theta_{i}}, \quad \partial_{i}=\frac{1}{2} e^{-\iota \theta_{i}}\left(\frac{\partial}{\partial \rho_{i}}-\frac{\iota}{\rho_{i}} \frac{\partial}{\partial \theta_{i}}\right)
$$

so that

$$
\begin{aligned}
H= & -\frac{1}{2} \sum_{i=1}^{N}\left(\frac{\partial^{2}}{\partial \rho_{i}^{2}}+\frac{1}{\rho_{i}} \frac{\partial}{\partial \rho_{i}}+\frac{1}{\rho_{i}^{2}} \frac{\partial^{2}}{\partial \theta_{i}^{2}}-\rho_{i}^{2}\right) \\
& -\frac{\alpha}{2} \sum_{i<j}\left[\frac{e^{-\iota \theta_{i}}\left(\frac{\partial}{\partial \rho_{i}}-\frac{\iota}{\rho_{i}} \frac{\partial}{\partial \theta_{i}}\right)-e^{-\iota \theta_{j}}\left(\frac{\partial}{\partial \rho_{j}}-\frac{\iota}{\rho_{j}} \frac{\partial}{\partial \theta_{j}}\right)}{\rho_{i} e^{-\iota \theta_{i}}-\rho_{j} e^{-\iota \theta_{j}}}\right. \\
& \left.-\frac{e^{\iota \theta_{i}}\left(\frac{\partial}{\partial \rho_{i}}+\frac{\iota}{\rho_{i}} \frac{\partial}{\partial \theta_{i}}\right)-e^{\iota \theta_{j}}\left(\frac{\partial}{\partial \rho_{j}}+\frac{\iota}{\rho_{j}} \frac{\partial}{\partial \theta_{j}}\right)}{\rho_{i} e^{\iota \theta_{i}}-\rho_{j} e^{\iota \theta_{j}}}\right] \\
+ & \frac{\alpha^{2}}{2} \sum_{i \neq j, k} \frac{1}{\left(\rho_{i} e^{-\iota \theta_{i}}-\rho_{j} e^{-\iota \theta_{j}}\right)\left(\rho_{i} e^{\iota \theta_{i}}-\rho_{k} e^{\iota \theta_{k}}\right)} .
\end{aligned}
$$

The eigenvalue equation is

$$
H \Psi\left(\rho_{i}, \theta_{i}\right)=E \Psi\left(\rho_{i}, \theta_{i}\right)
$$

The proposed "reduction" of dimensions is carried out at the level of this eigenvalue equation. This can be achieved naively in two possible ways: (a) by fixing the radial variables $\rho_{i}$ so that the equation depends only on the $\theta_{i}$ 's and the problem reduces to particles on a circle of fixed radius $\rho$ and (b) by keeping the angular variables $\theta_{i}$ constant, in which case the problem is only $\rho_{i}$-dependent and looks like particles on a line. We proceed to examine both these methods now. 


\section{Reduction of Free Anyons to a Circle}

Consider the eigenvalue equation for free anyons (no oscillator potential) at $\rho_{i}=\rho \forall i$. With no approximations whatever, we get

$$
\begin{aligned}
H \Psi= & -\left.\frac{1}{2} \sum_{i=1}^{N} \frac{\partial^{2} \Psi}{\partial \rho_{i}^{2}}\right|_{\rho_{i}=\rho}-\left.\frac{1}{2} \sum_{i=1}^{N} \frac{1}{\rho_{i}} \frac{\partial \Psi}{\partial \rho_{i}}\right|_{\rho_{i}=\rho} \\
& -\frac{\alpha}{2} \sum_{i<j}\left[\frac{1}{\rho_{i} e^{-\iota \theta_{i}}-\rho_{j} e^{-\iota \theta_{j}}}\left(e^{-\iota \theta_{i}} \frac{\partial}{\partial \rho_{i}}-e^{-\iota \theta_{j}} \frac{\partial}{\partial \rho_{j}}\right)\right. \\
& \left.-\frac{1}{\rho_{i} e^{\iota \theta_{i}}-\rho_{j} e^{\iota \theta_{j}}}\left(e^{\iota \theta_{i}} \frac{\partial}{\partial \rho_{i}}-e^{\iota \theta_{j}} \frac{\partial}{\partial \rho_{j}}\right)\right]\left.\Psi\right|_{\rho_{i}=\rho_{j}=\rho} \\
& -\frac{1}{2 \rho^{2}} \sum_{i} \frac{\partial^{2} \Psi}{\partial \theta_{i}^{2}}+\frac{\iota \alpha}{2 \rho^{2}}(N-1) \sum_{i} \frac{\partial \Psi}{\partial \theta_{i}} \\
+ & \frac{\alpha^{2}}{8 \rho^{2}} \sum_{i \neq j} \frac{1}{\sin ^{2}\left(\frac{\theta_{i j}}{2}\right)} \Psi+\frac{\alpha^{2}}{6 \rho^{2}} N(N-1)(N-2) \Psi .
\end{aligned}
$$

We wish to fix the radial coordinates so that the dynamics depends only on the angular variables and the problem is reduced to one dimension, viz the circle. To achieve this, one could naively drop the $\rho$-derivatives so that only the last two lines in the above equation survive and the Hamiltonian becomes, after some regrouping,

$$
H^{\prime}=\frac{1}{2 \rho^{2}}\left\{\sum_{i=1}^{N}\left[\iota \frac{\partial}{\partial \theta_{i}}+\alpha(N-1)\right]^{2}+\frac{\alpha^{2}}{4} \sum_{i \neq j} \frac{1}{\sin ^{2}\left(\frac{\theta_{i j}}{2}\right)}-\frac{\alpha^{2}}{3} N(N-1)(2 N-1)\right\}
$$

This is the Sutherland model, which is related to the Calogero model on a circle, but for the shifted momentum operator and the constant $\alpha^{2}$-dependent term that will just add to the eigenvalue. The shift can be annulled by performing a gauge transformation on the wave function:

$$
\Psi \rightarrow \Psi \exp \left(\iota \alpha(N-1) \sum_{i} \theta_{i}\right)
$$

This is the reduction obtained by Bhaduri and Li [11]. However, this model is not a reduced anyon model, because it rests on the over-restrictive condition that the wave functions be completely $\rho$-independent. (Nevertheless it could exist on its own right as some one-dimensional model, albeit with no anyonic connection!)

If we wish to retain the connection with the anyon system in such a dimensional reduction, the correct way to proceed is to evaluate the derivatives first and then equate all the radial variables to a fixed value $\rho$. Let us see how Eq (3.5) can be simplified. Now the $\rho_{i}$-derivatives in the $\alpha$-dependent term in will cancel if we 
consider the rather special case of wave functions that factor into radial and angular parts and with the radial part symmetric in the $\rho_{i}$ 's. In such a case, the Hamiltonian will look like

$$
H_{\text {red }}=-\frac{N}{2}\left[\frac{\partial^{2}}{\partial \rho^{2}}+\frac{1}{\rho} \frac{\partial}{\partial \rho}\right]+H^{\prime} .
$$

Thus the eigenvalue equation for this Hamiltonian is not that of the Sutherland model, but has inhomogeneous terms arising from the $\rho$-derivatives of the wave function. One could get rid of these terms in several ways: (a) One could demand that $\rho$-derivatives cancel each other. This could happen by choosing $\rho_{i}$-dependence of the wave functions to be of the form $\ln \rho_{i}$, appropriately symmetrised. However these may not be solutions to the original anyon problem. (b) One could evaluate the inhomogeneous terms for a fixed $\rho$ and choose only that value (if any) where these terms vanish. (c) Another possibility is to choose boundary conditions such that the contributions to the $\rho$-derivatives at the boundary cancel against the extra terms.

Suffice it to say that without additional restrictions, one does not obtain the Sutherland model or a related model on trying to restrict the anyon system to a circle.

\section{Reduction to a Line}

Another way of reducing the dimensions in the Hamiltonian (2.3) is to fix the angular variables and hence constrain the problem to a line. This also means that we wish to project out $\theta$-independent solutions of the anyon equation (2.2). Now the singular nature of the potential at $r_{i}=r_{j}$ demands that well-behaved wave functions be of the form $\Pi_{i<j}\left|r_{i j}\right|^{\alpha} \psi\left(r_{i}\right)$. Here we must look at the eigenvalue equation when all the $\theta_{i}$ 's are equated after evaluating the angular derivatives on the wave function. Since the total angular momentum $J=\iota \sum_{i=1}^{N} \frac{\partial}{\partial \theta_{i}}$ is conserved, we can look at solutions for which the angular dependence of the wave function can be separated as

$$
\Psi\left(\rho_{i}, \theta_{i}\right)=\sum_{\left\{n_{i}\right\}} \exp \left(\iota \Sigma_{i} n_{i} \theta_{i}\right) \psi_{n_{i}}\left(\rho_{i}\right)
$$


where $\sum_{i} n_{i}=j$ is the total angular momentum eigenvalue. The eigenvalue equation becomes

$$
\sum_{\left\{n_{i}\right\}} H_{n_{i}} \psi_{n_{i}}\left(\rho_{i}\right)=E \sum_{\left\{n_{i}\right\}} \psi_{n_{i}}\left(\rho_{i}\right)
$$

where

$$
\begin{aligned}
H_{n_{i}}= & -\frac{1}{2} \sum_{i=1}^{N}\left(\frac{\partial^{2}}{\partial \rho_{i}^{2}}+\frac{1}{\rho_{i}} \frac{\partial}{\partial \rho_{i}}-\frac{n_{i}^{2}}{\rho_{i}^{2}}\right)+\frac{1}{2} \sum_{i=1}^{N} \rho_{i}^{2} \\
& -\frac{\alpha}{2} \sum_{i<j}\left[\frac{e^{-\iota \theta_{i}}\left(\frac{\partial}{\partial \rho_{i}}+\frac{n_{i}}{\rho_{i}}\right)-e^{-\iota \theta_{j}}\left(\frac{\partial}{\partial \rho_{j}}+\frac{n_{j}}{\rho_{j}}\right)}{\rho_{i} e^{-\iota \theta_{i}}-\rho_{j} e^{-\iota \theta_{j}}}\right] \\
& \left.-\frac{e^{\iota \theta_{i}}\left(\frac{\partial}{\partial \rho_{i}}-\frac{n_{i}}{\rho_{i}}\right)-e^{\iota \theta_{j}}\left(\frac{\partial}{\partial \rho_{j}}-\frac{n_{j}}{\rho_{j}}\right)}{\rho_{i} e^{\iota \theta_{i}}-\rho_{j} e^{\iota \theta_{j}}}\right] \\
+ & \frac{\alpha^{2}}{2} \sum_{i \neq j, k} \frac{1}{\left(\rho_{i} e^{-\iota \theta_{i}}-\rho_{j} e^{-\iota \theta_{j}}\right)\left(\rho_{i} e^{\iota \theta_{i}}-\rho_{k} e^{\iota \theta_{k}}\right)} .
\end{aligned}
$$

In contrast to the previous case, we have gotten rid of the $\theta$-derivatives without the complication of inhomogeneous terms. But now the eigenvalue equation involves a sum over all possible sets $\left\{n_{i}: \Sigma_{i} n_{i}=j\right\}$ and in complete generality, involves considering infinite sets of $n_{i}$ 's. The problem becomes hard to tackle because of this. If, for simplicity, we choose just one set, the the sum collapses to one term. (We must keep in mind the restrictions imposed by this choice.) On equating all the angular variables, we get the Hamiltonian for anyons restricted to a line as

$$
\begin{aligned}
H_{n_{i}}= & -\frac{1}{2} \sum_{i=1}^{N}\left(\frac{\partial^{2}}{\partial \rho_{i}^{2}}+\frac{1}{\rho_{i}} \frac{\partial}{\partial \rho_{i}}-\frac{n_{i}^{2}}{\rho_{i}^{2}}\right)+\frac{1}{2} \sum_{i=1}^{N} \rho_{i}^{2} \\
& -2 \alpha \sum_{i<j} \frac{n_{i} \rho_{j}-n_{j} \rho_{i}}{\rho_{j} \rho_{i}\left(\rho_{i}-\rho_{j}\right)}+\frac{\alpha^{2}}{2} \sum_{i \neq j} \frac{1}{\left(\rho_{i}-\rho_{j}\right)^{2}} .
\end{aligned}
$$

If we now choose $n_{i}=0 \forall i$, the Hamiltonian simplifies to that of the standard CSM but for the $\frac{1}{\rho_{i}} \frac{\partial}{\partial \rho_{i}}$ term. This can easily be brought to the standard CSM form by taking $\psi_{0}\left(\rho_{i}\right)=\prod_{i} \rho_{i}^{-1 / 2} \chi\left(\rho_{i}\right)$. This scaling is also consistent with that required of the inner product when reducing the dimensions to one, i.e ensures that $\int \rho d \rho\left|\psi_{0}\right|^{2} \rightarrow \int d \rho|\chi|^{2}$. In the process, however, an extra potential is generated. $\chi\left(\rho_{i}\right)$ is then an eigenfunction of the Hamiltonian

$$
H_{\text {red }}=-\frac{1}{2} \sum_{i} \frac{\partial^{2}}{\partial \rho_{i}^{2}}+\frac{\alpha^{2}}{2} \sum_{i \neq j} \frac{1}{\left(\rho_{i}-\rho_{j}\right)^{2}}+\frac{1}{2} \sum_{i}\left(\rho_{i}^{2}-\frac{1}{4 \rho_{i}^{2}}\right)
$$


which is the CSM Hamiltonian [3] (in an oscillator confinement) but with two noteworthy differences: (a) the additional attractive $\frac{1}{8 \rho_{i}^{2}}$ potential. (b) the range of the variable $\rho_{i}$ being $(0, \infty)$, this model is defined on the half-line.

When seeking solutions to this model from those of the original anyon system, we notice that the wave functions, that contain the factor $\Pi_{i} \rho_{i}^{-1 / 2}$, can no longer be factorised as in (4.8) for a finite sum over the $n_{i}$ 's. Therefore this model no longer has a direct bearing on the anyon system. To stay within the purview of anyonic solutions, one must first take out this factor and then reduce. This will be explored in the sub-section that follows.

However, the model (4.12) is interesting in its own right. Note that a CSM model modified by a term of the form $\frac{a}{\rho_{i}} \frac{\partial}{\partial \rho_{i}}$ can be brought to the standard CSM form by redefining the wave function. Now such a term appears in the radial part of the Laplacian in $d$ dimensions:

$$
\nabla^{2}=\frac{\partial^{2}}{\partial \rho^{2}}+\frac{d-1}{\rho} \frac{\partial}{\partial \rho}+\nabla_{\perp}^{2}
$$

where $\nabla_{\perp}$ is the part of the Laplacian orthogonal to the radial part. On pulling out a factor $\rho^{-d / 2}$ from the wave function, the radial part reduces to

$$
\nabla_{\rho}^{2}=\frac{\partial^{2}}{\partial \rho^{2}}-\frac{(d-1)(d-3)}{4 \rho^{2}}
$$

generating an extra potential. This scaling is also required of the inner product when reducing the dimensions. Note that the extra potential term is attractive for $d=2$, absent for $d=3$ and repulsive for higher dimensions. Thus if our model is spherically symmetric, then the spherically invariant eigenfunctions satisfy the eigenvalue equation

$$
-\frac{1}{2} \nabla_{\rho}^{2} \psi(\rho)=E \psi(\rho)
$$

Now a potential of the kind $V\left(\mathbf{r}_{i}\right)=\sum_{i \neq j, k=1}^{N} \frac{\mathbf{r}_{i j} \cdot \mathbf{r}_{i k}}{r_{i j}^{2} r_{i k}^{2}}$, when restricted to a line (fix all angular variables) yields the standard CSM inverse-square two-body interaction. Therefore we see that there is a family of higher dimensional models that can be reduced to a CSM model with a $\frac{1}{\rho_{i}^{2}}$ potential defined for $\rho>0$, that is therefore important to analyse. 


\subsection{Anyon Solutions and a Related Model}

We will now explore a way of effecting a reduction while remaining within the realm of solutions of the anyon equation (2.2).

It is well known (see [2]) that exact solutions for the anyon eigenvalue equation (2.3) are of the form

$$
\Psi\left(\rho_{i}, \theta_{i}\right)=\prod_{i<j}\left|\mathbf{r}_{i}-\mathbf{r}_{j}\right|^{\alpha} \exp \left(-\frac{1}{2} \sum_{i} \rho_{i}^{2}\right) \psi\left(r_{i}, \theta_{i}\right) .
$$

But here, the $\theta$-dependence of the wave function cannot be factorised as in eq. (4.8) if as in the previous section on chooses only one set of $n_{i}$ 's and not the infinite sum.

Now the singular nature of the anyonic potential at $\mathbf{r}_{i}=\mathbf{r}_{j}$ demands that wellbehaved wave functions be of the form $\Pi_{i<j}\left|\mathbf{r}_{i j}\right|^{\alpha} \psi\left(r_{i}\right)$. Therefore, one must carry out the reduction after extracting this factor from the wave function in eq. (2.4).

$\psi\left(r_{i}, \theta_{i}\right)$ then satisfies the eigenvalue equation of the Hamiltonian

$$
\begin{aligned}
H= & -\frac{1}{2} \sum_{i=1}^{N}\left(\frac{\partial^{2}}{\partial \rho_{i}^{2}}+\frac{1}{\rho_{i}} \frac{\partial}{\partial \rho_{i}}+\frac{1}{\rho_{i}^{2}} \frac{\partial^{2}}{\partial \theta_{i}^{2}}-\rho_{i}^{2}\right) \\
& -\alpha \sum_{i<j} \frac{e^{-\iota \theta_{i}}\left(\frac{\partial}{\partial \rho_{i}}-\frac{\iota}{\rho_{i}} \frac{\partial}{\partial \theta_{i}}\right)-e^{-\iota \theta_{j}}\left(\frac{\partial}{\partial \rho_{j}}-\frac{\iota}{\rho_{j}} \frac{\partial}{\partial \theta_{j}}\right)}{\rho_{i} e^{\iota \theta_{i}}-\rho_{j} e^{\iota \theta_{j}}}
\end{aligned}
$$

Here if we look for $\theta$-dependence of the form (4.8) and choose $n_{i}=0 \forall i$, the $\theta$ dependence drops out and $\chi\left(\rho_{i}\right)$ satisfies the eigenvalue equation of the Hamiltonian

$$
H=-\frac{1}{2} \sum_{i=1}^{N}\left(\frac{\partial^{2}}{\partial \rho_{i}^{2}}+\frac{1}{\rho_{i}} \frac{\partial}{\partial \rho_{i}}-\rho_{i}^{2}\right)-\alpha \sum_{i<j} \frac{\partial_{i j}}{\rho_{i j}}
$$

(Here the subscript $i j$ means $X_{i j}=X_{i}-X_{j}$.) We can now put back the factor $\left|\rho_{i j}\right|^{-\alpha}$ (which has no $\theta$-dependence) into this, and also take out the factor $\Pi_{i} \rho_{i}^{-1 / 2}$ so that the reduced Hamiltonian is

$$
H=-\frac{1}{2} \sum_{i=1}^{N} \frac{\partial^{2}}{\partial \rho_{i}^{2}}+\frac{1}{2} \sum_{i}\left(\rho_{i}^{2}-\frac{1}{4 \rho_{i}^{2}}\right)+\frac{\alpha^{2}}{2} \sum_{i \neq j} \frac{1}{\left(\rho_{i}-\rho_{j}\right)^{2}}+\frac{\alpha}{2} \sum_{i<j} \frac{1}{\rho_{i} \rho_{j}} .
$$

We now have a model similar to the earlier one (4.12) except for the new $\alpha$-dependent term. This is a general inverse quadratic potential in the $\rho$ coordinates, which has solutions obtained from the anyon model we started out with. The solutions we need are those corresponding to the condition $n_{i}=0 \forall i$, which are the $j=0$ solutions. 
The wave functions $\chi_{0}$ are now functions of the variable $t=\sum_{i} \rho_{i}^{2}$ and satisfy the confluent hyper-geometric equation (see [2]) with the polynomial solution

$$
\chi_{0}(t)=M\left(-m, N+\alpha \frac{N(N-1)}{2}, t\right)
$$

and normalizability requires that energy is quantized as

$$
E=N+2 m+\alpha \frac{N(N-1)}{2} .
$$

\section{Summary and Conclusion}

In this work the dimensional reduction of the $\mathrm{N}$-anyon system to a circle and to a line has been studied from a mathematical point of view. It must be noted that our philosophy is slightly different from previous works along these lines, for instance ref

[6], where the physical problem of particles with exchange statistics in one dimension is dealt with. Here we take the standard two-dimensional anyon eigenvalue equation and project it onto one dimension in two ways. The resultant model not only carries an anyonic flavour but also has solutions projected out of the two-dimensional anyon solutions.

Reduction to a circle, if carried out in a naive way by simply dropping the radial derivatives, leads to the Sutherland model, as obtained in [11] (This model seems to arise in connection with edge states in the composite fermion model for the quantum Hall effect [12]). This process however does not retain connection with the original anyon model, since it means imposing restrictions on the wave functions that are too severe. One tries to retain the connections to this exactly solvable model for some particular class of solutions to the anyon problem if the radial derivatives are evaluated first and then chosen so as to cancel. A related model with extra potential terms can be obtained if the radial terms evaluated at a fixed value of the radial variables are retained.

Dimensional reduction of the the N-anyon system to a (half-)line, obtained by requiring the $\theta$-dependence of the wave functions to factor out and summing over one set out of the infinite possible sets $n_{i}$, yields a model related to the CalogeroSutherland model. This model is important from the relation it bears to reduction of 
higher dimensional rotationally invariant models with CSM-like interactions. However, this model does not have solutions that can be adapted from the known exact solutions for anyons. This is because the factor $\Pi_{i<j}\left|\mathbf{r}_{i j}\right|^{\alpha}$ in anyonic solutions cannot be factored as in (4.8) for a single set of $n_{i}$ s. So one carries out the reduction after taking out this factor. The model now obtained does have solutions reduced from the anyon solutions. These are not the only solutions to the model and means of obtaining other solutions to this problem are under investigation.

One therefore concludes that the anyon system cannot be directly reduced (in our sense of the term "reduction") to one dimension to give the CSM .

One also concludes that the reduction procedure does not give a way to deduce solutions to the original anyon model in the regime of eigenvalues having non-linear dependence on $\alpha$. This is because these solutions require considering infinite sums over the $n_{i}$ 's (see ref. [2]) and here one loses the connection to the CSM-like model.

\section{Acknowledgments}

I thank M. V. N. Murthy for suggesting this line of research and for extremely fruitful discussions. The results obtained here are a product of extensive discussions with G. Date, for which I am indebted to him. I am also thankful to both of them for going through the manuscript carefully and offering useful suggestions.

\section{References}

[1] Abundant literature is now available on the subject of anyons and solutions, starting with the pioneering paper, J. M. Leinaas and J. Myrheim, Il Nuovo Cimento B37 (1977) 1 , G. A. Goldin, R. Menikoff and D. H. Sharp, J. Math. Phys. 21 (1980) 650, J. Math. Phys.22 (1981) 1664, and F. Wilczek, Phys. Rev. Lett. 48 (1982) 1144, Phys. Rev. Lett. 49 (1982) 957, A. Lerda, Anyons, Lecture Notes in Physics, m14 is a recent monograph.

[2] G. Date, M. Krishna and M. V. N. Murthy, Int. J. Mod. Phys. A 9 (1994) 2545 (contains useful discussions and references for anyons). 
[3] F. Calogero, J. Math. Phys. 10 (1969) 2197, J. Math. Phys. 12 (1971) 419.

[4] B. Sutherland, J. Math. Phys. 12 (1971) 246, J. Math. Phys. 12 (1971) 251, Phys. Rev. A4 (1971), Phys. Rev. A5 (1971) 1372.

[5] V. Pasquier, "A lecture on the Calogero-sutherland Models", SACLAY-SPHT94-060, hep-th/9405104.

[6] T. H. Hansson, J. M. Leinaas and J. Myrheim, Nucl. Phys. B 384 (1992) 559.

[7] A. P. Polychronakos, Nucl. Phys B324 (1989) 597, Phys. Lett. B264 (1991) 362.

[8] F. D. M. Haldane, Phys. Rev. Lett. 67 (1991) 937.

[9] For the connection between the Calogero-Sutherland model and fractional exchange statistics, see M. V. N. Murthy and R. Shankar, Phys. Rev. Lett. 72 (1994) 604, Z. N. C. Ha, Nucl. Phys. B435 [FS] (1995) 604 and the references therein.

[10] U. Aglietti, L. Griguolo, R. Jackiw, S.-Y. Pi, Phys. Rev. Lett. 77 (1996) 4406, I. Andrić, V. Bardek and L. Jonke, hep-th/9507110.

[11] R. K. Bhaduri and S. Li, cond-mat/9404068.

[12] Y. Yu and Z. Zhu, cond-mat/9704124. 